\title{
Ce que les vibrisses disent au cerveau tactile
}

> Le système des vibrisses des rongeurs est devenu un des modèles principaux pour l'étude des propriétés fonctionnelles des neurones sensoriels. Ceci est dû, d'une part, à la structure très bien connue des voies afférentes qui relient les mécanorécepteurs à la base des vibrisses au cortex somatosensoriel primaire et, d'autre part, à l'accessibilité des senseurs tactiles permettant de contrôler l'entrée sensorielle à l'échelle du micron et de la milliseconde. L'observation de l'utilisation des vibrisses par le rongeur indique que les contacts avec des objets et des textures se font avec des dizaines de vibrisses simultanément. Nous avons exploré le codage neuronal dans le cortex à tonneaux, le cortex qui reçoit les informations depuis les vibrisses. En combinant enregistrements multiélectrodes et stimulation tactile multivibrissales avec une analyse théorique, nous avons mis en évidence que plusieurs types de réponses neuronales, semblables à celles décrites dans des aires différentes du système visuel, coexistent dans le même volume cortical. Ceci indique que des schémas de codage variés peuvent être implémentés dans le même réseau cortical et seraient mis en jeu pour analyser au mieux les stimulations tactiles diverses et changeantes auxquelles sont confrontés les rongeurs dans leur environnement naturel. <

\section{Plus d'une voie nous mène de la sensation à la perception}

Nous avons l'impression prégnante de percevoir le monde de manière directe et explicite. Cependant, l'analyse des informations atteignant les récepteurs périphériques par différentes modalités sensorielles

Vignette (๔ Photo Jean Livet).

\author{
Luc Estebanez ${ }^{1,2}$, Sami El Boustani ${ }^{1,3}$, \\ Alain Destexhe ${ }^{1}$, Daniel $\varepsilon$. Shulz ${ }^{1}$
}

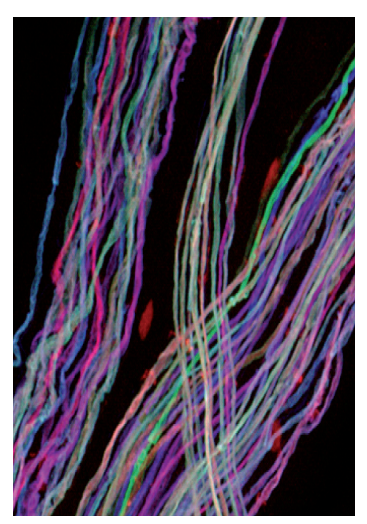

${ }^{1}$ Unité de neurosciences, information et complexité (UNIC), Centre national de la recherche scientifique, 1 avenue de la Terrasse, bâtiment 32-33, 91190 Gif sur Yvette, France ; ${ }^{2}$ Actuellement au MaxDelbrück-centrum für molekular medizin, Berlin, Allemagne; ${ }^{3}$ Actuellement au Picower institute for learning and memory, department of brain and cogni-

peut conduire à une perception déformée, très différente d'un reflet immédiat et homothétique de la réalité. L'étude des illusions sensorielles, à savoir les perceptions qui diffèrent de la réalité physique, particulièrement décrites dans le système visuel, nous tive sciences, Massachusetts institute of technology, Cambridge MA, États-Unis.

L. Estebanez et S. El Boustani ont contribué à parts égales à ce travail.

shulz@unic.cnrs-gif.fr renseigne sur les relations complexes entre réalité et perception. L'école de la Gestalt ${ }^{1}$, dans son entreprise d'explication phénoménologique de la perception, propose pour comprendre ces illusions un certain nombre de principes clés parmi lesquels la «réification », qui stipule qu'un percept contient une information spatiale plus riche que le stimulus sensoriel sur lequel ce percept est fondé. Cet enrichissement des percepts par rapport aux stimulus élémentaires qui atteignent nos sens est régi par une série de règles dites de « groupement perceptif ». Par exemple la règle de « continuité » prédit - de façon exacte - l'émergence d'un percept en mouvement continu à partir de la présentation successive d'un même stimulus élémentaire statique à différents endroits du champ visuel, suivant une trajectoire virtuelle définie (Figure lA). Même si, à chaque instant, l'image visuelle est statique, le sujet a une perception de mouvement continu de l'objet le long de cette trajectoire ou chemin d'association. Une autre règle classique de groupement perceptif est la règle de «clôture », qui est illustrée par l'illusion du Triangle de Kanizsa (Figure 1B): les contours illusoires d'un triangle accompagnés de la perception d'une surface triangulaire plus brillante que le fond sont clairement perçus même si ni ces contours, ni la surface du triangle, ne font partie explicitement de l'image.

${ }^{1}$ École de psychologie de la perception qui trouve son origine au début du xx siècle en Allemagne. 
A

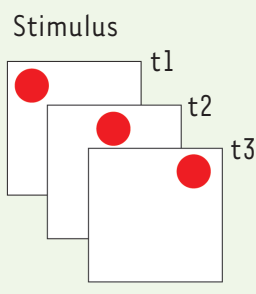

C
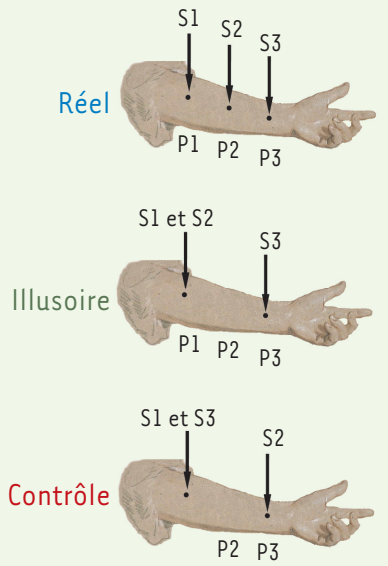
Perception

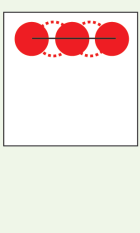

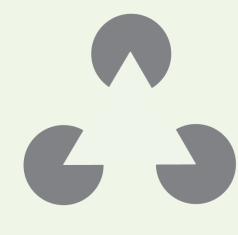

D

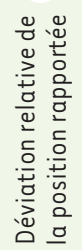

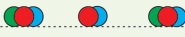

S1 $\quad$ S2 $\quad$ s3

Ordre de simulation

$\varepsilon$

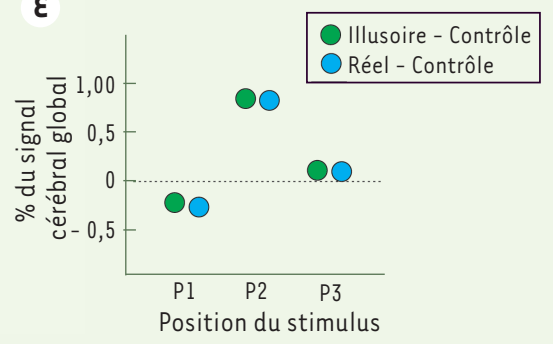

Figure 1. Exemples d'illusions visuelles et tactiles.

A. Illusion de mouvement apparent. Un point est présenté de manière statique successivement à trois endroits du champ visuel. La perception qui en résulte est celle d'un mouvement continu du point le long d'une trajectoire virtuelle. $B$. Triangle de Kanizsa. La surface triangulaire qui se dégage de l'image n'a pas de réalité physique. C. Illusion tactile saltatoire. Des stimulus tactiles ponctuels sont délivrés à trois endroits de l'avant-bras ( $\mathrm{Pl}$, $P 2$ et $P 3$ ). Les points stimulés ( $S 1, S 2$ et $S 3$ ) dans chacune des trois séquences de stimulation diffèrent. Dans le cas «réel», les trois points sont stimulés séquentiellement; dans le cas «illusoire », les deux premiers stimulus sont appliqués en position $1(\mathrm{Pl})$ et le troisième en position 3 (P3) ; dans le cas «contrôle», les stimulus 1 et 3 sont appliqués en position 1 (Pl) et le stimulus 2 est appliqué en position 3 (P3). Dans les deux premiers cas, le sujet rapporte une sensation de touché localisé dans la position 2, ce qui n'est pas le cas pour la situation contrôle. Dessin d'après Louis Tocque (xvIII ${ }^{\mathrm{e}}$ siècle, Le Louvre, Paris, France). $D$. Mesure de la déviation relative de la localisation rapportée par le sujet par rapport à la position réelle du stimulus en fonction de l'ordre de stimulation pour les trois cas présentés en $C$.

Notez dans le cas « illusoire » (point vert) que le sujet rapporte une sensation tactile dans la position P2 à mi-chemin entre P1 et P3, alors qu'aucun stimulus n'a été délivré à cet endroit. Adapté de [23]. $\varepsilon$. Étude par IRM fonctionnelle des zones d'activation corticale dans les trois conditions décrites en $C$. Le pourcentage du signal global dans l'aire de représentation de la position 2 dans le cortex somatosensoriel primaire chez le sujet humain relatif à la situation contrôle est donné en fonction de la position du stimulus. Notez l'activation corticale comparable pour la position P2 dans le cas «illusoire» (points verts) et le cas « réel » (points bleus). Adapté de [2].

De nombreuses autres illusions visuelles, semblables à celle du mouvement apparent décrite ci-dessus, permettent d'affirmer que les mécanismes neurophysiologiques sous-jacents aux perceptions visuelles, loin d'effectuer une transmission de l'information périphérique par lignes dédiées et une simple sommation, réalisent une analyse nécessairement non linéaire des afférences sensorielles, comme par exemple la complétion d'une information manquante dans l'image ou l'extrapolation de la position d'un objet en mouvement.

Tout comme dans la modalité visuelle, la perception tactile s'appuie sur un traitement non linéaire et hautement dynamique de l'information sensorielle acquise au travers des mécanorécepteurs de la peau. Ces non-linéarités sont particulièrement évidentes dans le cas des illusions tactiles, comme celle connue sous le nom du «lapin qui saute» [1]. Dans cette illusion, le touché très rapproché dans le temps à deux endroits de la peau, par exemple à deux endroits de l'avant-bras, crée une sensation de touchés successifs intermédiaires entre les deux points stimulés. Ainsi, si on touche d'abord la peau près du coude et ensuite près du poignet, le sujet perçoit des touchés entre ces deux points comme si un lapin sautait du coude au poignet en passant par des points intermédiaires, même si aucun stimulus physique n'a été présenté entre ces deux points (Figure $2 C-D$ ). II a été montré par imagerie par résonance magnétique fonctionnelle (IRMf) que lors de l'illusion tactile, le cortex somatosensoriel primaire controlatéral, qui reçoit les informations tactiles depuis la périphérie, est activé dans des zones de représentation qui correspondent aux zones perçues, mais non stimulées. L'activation corticale correspond donc davantage à la sensation perçue plutôt qu'au stimulus physique délivré par l'expérimentateur (Figure 2E) [2]. Récemment, une variante de cette illusion a été décrite dans laquelle le «lapin qui saute» est perçu sur un bâton maintenu entre les deux index des deux mains, suggérant que les bases neuronales de cette illusion reposent certes sur la représentation somatotopique du corps, comme nous l'avons vu plus haut dans le travail de Blankenburg et al. [2], mais également sur des représentations extracorporelles [3].

Ces quelques exemples d'illusions visuelles et tactiles nous renseignent sur l'existence d'effets contextuels de 


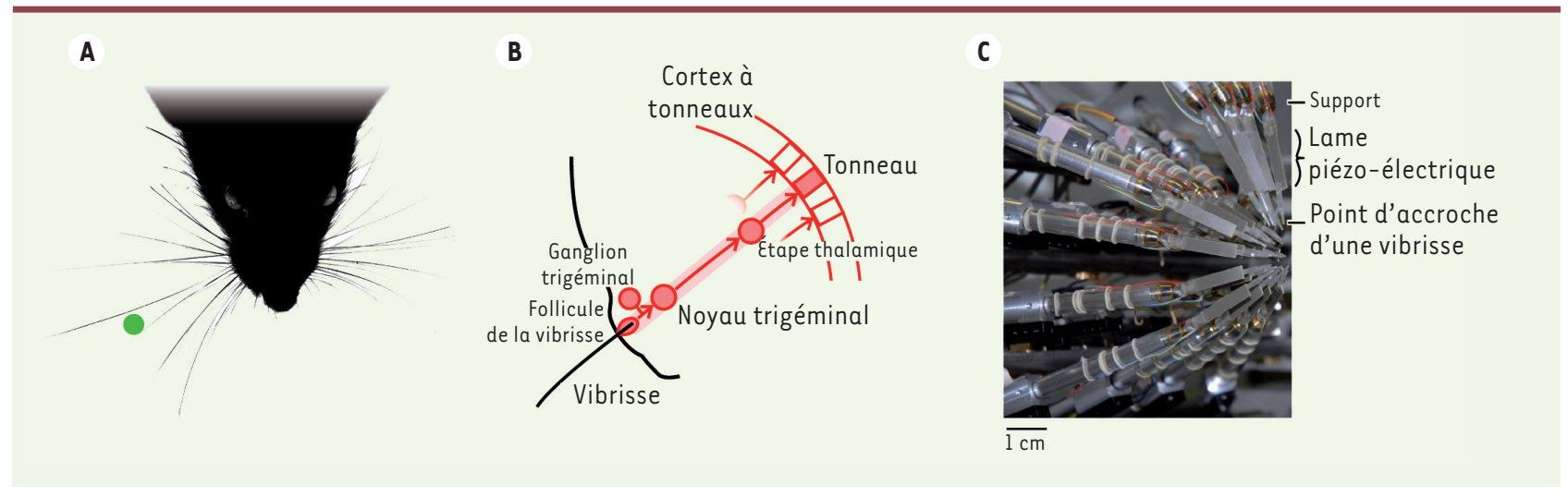

Figure 2. Le système vibrissal. A. Les nombreuses vibrisses distribuées en éventail autour du museau (face dorsale) effectuent des mouvements de balayage rythmique durant la détection d'un objet (en vert). B. Modèle feed forward du système vibrissal. Chaque vibrisse est un poil inerte enchâssé dans un follicule contenant des mécanorécepteurs. Les déflexions de la vibrisse sont traduites en potentiels d'action propagés le long d'une chaîne de noyaux sous-corticaux pour aboutir au cortex à tonneaux controlatéral. De nombreuses connexions latérales ou en arrière (feedback) entre les constituants du système sont connues (non représentées ici), mais la compréhension des réponses fonctionnelles des neurones du cortex à tonneaux s'appuie essentiellement sur le simple modèle feed forward. C. Matrice de stimulateurs de vibrisses développée dans le laboratoire afin d'explorer systématiquement les réponses neuronales à des stimulations multivibrissales complexes. Chaque vibrisse attachée à une lame piézo-électrique peut être déplacée latéralement jusqu'à 100 fois par seconde [19].

la stimulation sensorielle et, donc, de mécanismes neuronaux complexes mis en jeu par des interactions entre le signal afférent induit par la stimulation physique et des signaux neuronaux générés par le système nerveux de façon intrinsèque. Par conséquent, pour comprendre la relation entre une perception illusoire et la réalité physique, il est nécessaire d'accéder à l'organisation anatomo-fonctionnelle du système et, en particulier, d'étudier l'activité électrique des neurones concernés en fonction des entrées sensorielles. Le cortex somatosensoriel des rongeurs se prête particulièrement bien à l'étude de cette question, et il est devenu le modèle prédominant en neurophysiologie pour l'étude du codage de l'information tactile.

\section{Le système de vibrisses du rongeur : un modèle de choix pour l'analyse multi-échelle de la sensation tactile}

Les rongeurs, et en particulier le rat, explorent leur environnement à l'aide de mouvements oscillatoires de protraction et de rétraction des vibrisses situées de part et d'autre du museau, ce qui permet à l'animal d'analyser tactilement les objets proches [4]. Lors des contacts des vibrisses avec les objets environnants, l'information tactile relayée au système nerveux central permet au rat de déterminer sa position par rapport aux objets et d'obtenir des informations sur leur forme et texture.

Un large volume cortical est dédié à la représentation et au traitement des informations sensorielles en provenance des vibrisses. Spécifiquement, la région postéro-médiane du cortex somatosensoriel primaire, appelée aussi cortex à tonneaux (barrel cortex), est l'aire corticale qui reçoit l'information sensorielle en provenance des vibrisses faciales. Elle contient une carte somatotopique des vibrisses telle qu'à chaque vibrisse correspond une unité cyto-architectonique distincte dans la couche IV [5]. Ainsi, il existe une matrice de tonneaux dans le cortex correspondant à la matrice mystaciale ${ }^{2}$ périphérique $[6,7]$. De ce fait, le cortex à tonneaux a été classiquement considéré comme une juxtaposition de colonnes anatomo-fonctionnelles indépendantes, et la voie depuis les vibrisses comme un système composé de lignes de traitement indépendantes. Les contacts des nombreuses vibrisses du rat sur les objets $[8,9]$ (Figure 2A) seraient traduits en activité neuronale et relayés séparément pour chaque vibrisse par une série de noyaux successifs, avant d'aboutir dans une zone du cortex primaire somatosensoriel où à chaque vibrisse correspond un tonneau qui lui est essentiellement dédié (Figure 2B).

Cependant, des études électrophysiologiques intracellulaires dans plusieurs laboratoires américains [10, 11], ainsi que dans le nôtre [12], et des études établissant des cartes d'activation corticale à l'aide d'enregistrements multisites simultanés [13, 14], ont montré que l'information convergente sur un neurone cortical est, comme dans le système visuel [15], très étendue, et provient de plusieurs vibrisses au-delà du centre du champ récepteur. Cette étendue spatio-temporelle du champ récepteur sous liminaire suggère qu'à chaque étape du système vibrissal, un réseau dynamique de connexions latérales et rétrogrades (non représentées sur la Figure $2 B$ ), fournissent un substrat potentiel pour des interactions temporelles complexes entre les

${ }^{2}$ La matrice mystaciale est constituée de l'ensemble des vibrisses des rongeurs organisées sur leur museau en rangées et en arcs selon des positions fixes. 
informations en provenance de plusieurs vibrisses simultanément ou en léger décalage temporel. Ainsi, les neurones du cortex à tonneaux répondent préférentiellement à certaines séquences spatio-temporelles de stimulations multivibrissales impliquant de nombreuses vibrisses en s'écartant fortement des prédictions d'un simple modèle linéaire [7, 16-18].

\section{Intégration contextuelle de l'information tactile}

La description du système des vibrisses-cortex à tonneaux a été classiquement réalisée avec des stimulus ponctuels délivrés sur une ou quelques vibrisses à la fois. Cependant, l'étude de l'intégration des informations multivibrissales nécessite de pouvoir stimuler plusieurs, voire des dizaines de vibrisses simultanément et indépendamment. Nous avons développé une matrice de stimulation composée de 25 stimulateurs piézo-électriques indépendants adaptés aux cinq arcs et cinq rangées des vibrisses faciales [19] (Figure 2C). Ce stimulateur permet de délivrer de manière contrôlée des stimulus tactiles mimant de véritables scènes tactiles étendues, spatialement et temporellement. Ces scènes sont particulièrement à même de susciter des réponses non linéaires des neurones du cortex à tonneaux.

En particulier, nous avons testé des scènes tactiles virtuelles composées d'une stimulation identique pour chaque vibrisse (une déflexion caudale), mais qui diffèrent dans leur modèle spatial multivibrissal (déflexion successive de chaque vibrisse). Cette vague de déflection mime l'effet d'une barre se déplaçant à vitesse constante dans le champ des vibrisses, suivant huit directions différentes. Nous avons ainsi pu mesurer la sélectivité des neurones du cortex à tonneaux pour ces différentes directions. Nous avons trouvé qu'une majorité de neurones montrent une sélectivité marquée pour une direction donnée de déplacement de la barre. Cette sélectivité est probablement soustendue par des connexions intracorticales latérales $[18,20]$.

Ainsi, au contraire de la vision classique linéaire de l'organisation du système vibrisse-cortex à tonneaux, le cortex semble intégrer simultanément l'information tactile sur la totalité de la matrice mystaciale des vibrisses, assurant ainsi l'implémentation d'une analyse contextuelle de l'information tactile.

Pour établir un nouveau modèle du traitement cortical des stimulations des vibrisses incluant ces aspects non linéaires, nous avons soumis des rats anesthésiés à des stimulations mécaniques contrôlées (un bruit blanc jusqu'à $90 \mathrm{~Hz}^{3}$ ) d'un grand nombre de vibrisses (Figure 2C) [21]. Nous nous sommes intéressés à deux scènes de stimulation : dans un cas, toutes les vibrisses reçoivent une stimulation identique (stimulation en bruit blanc corrélée), tandis que, dans le second cas, les vibrisses reçoivent chacune une stimulation différente (stimulation « décorrélée »). Contrairement à ce qui est prédit par le modèle classique linéaire de la réponse du cortex à tonneaux, nous avons observé que les neurones corticaux réagissent de façon très contrastée à ces deux types de stimulations. Spécifiquement, en utilisant une méthode

${ }^{3}$ On désigne ainsi un bruit qui s'étend uniformément sur toutes les fréquences (comme la lumière blanche).
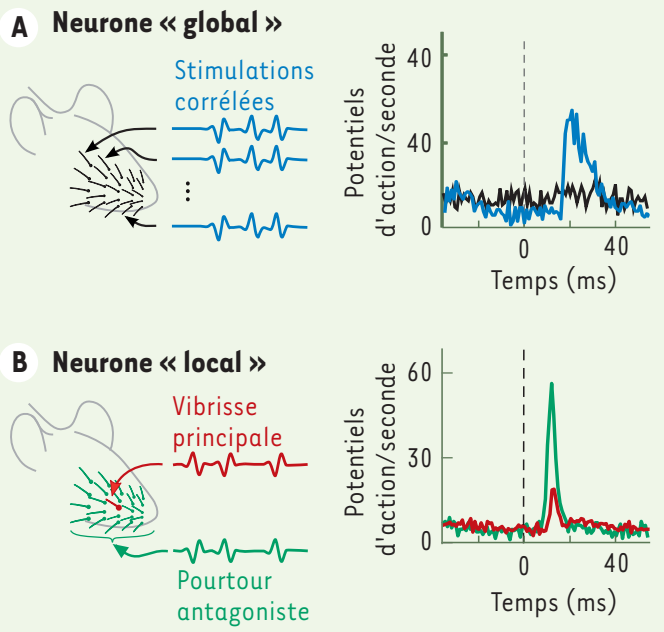

Figure 3. Deux classes de neurones sélectifs pour des caractéristiques multivibrissales des stimulations. $\boldsymbol{A}$. Histogramme de probabilité de décharge neuronale lorsque toutes les vibrisses sont stimulées de façon identique (gauche). Un neurone « global» répond à chaque stimulation par une décharge de potentiels d'actions significative (trace bleue), mais il ne répond pas du tout si les temps de stimulation de chaque vibrisse sont «décorrélés » les uns des autres (trace noire). B. Exemple d'un neurone «local » qui préfère une stimulation opposée de la vibrisse principale (en rouge, vibrisse correspondant au tonneau dans lequel on enregistre l'activité neuronale) contre toutes les vibrisses périphériques (en vert). Le neurone «local » répond davantage à une stimulation opposée (antagoniste) de la vibrisse principale et de la périphérie (trace verte) qu'à une stimulation uniquement de la vibrisse principale (trace rouge) (adapté de [21]).

qui permet d'identifier les stimulations les plus efficaces pour déclencher une réponse neuronale (I'analyse en corrélation inverse, voir Encadré 1), nous avons pu montrer l'existence d'une population de neurones qui répondent spécifiquement aux stimulations corrélées, et pas aux stimulations décorrélées: les neurones « globaux (Figure 3A).

Au-delà de l'observation de ce nouveau type cellulaire « global», nous avons également étudié l'impact du changement de corrélation sur le type cellulaire classiquement décrit dans le cortex à tonneaux, c'est-à-dire les cellules «locales». Ces dernières sont connues pour répondre spécifiquement aux stimulations de la vibrisse principale, soit la vibrisse alignée sur le tonneau dans lequel le neurone se trouve (Figure 2B). Durant les stimulations décorrélées, c'est la réponse à la vibrisse principale que nous avons observée. Cependant, lorsque les 25 vibrisses sont corrélées, la réponse 


\section{ANALYSE PAR CORRÉLATION INVERSE}

L'analyse par corrélation inverse (reverse correlation analysis) consiste en une estimation des relations statistiques entre le stimulus et la réponse neuronale évoquée. Dans le cas le plus simple, lorsque le stimulus est décrit par l'évolution d'une seule variable au cours du temps (par exemple la pression de l'air dans le cas du son), on calcule la corrélation entre la réponse neuronale en moyennant les segments de stimulus qui précèdent immédiatement chaque potentiel d'action (PA) émis par le neurone enregistré. Cette technique, connue sous le nom de spike-triggered average (STA), révèle la partie du stimulus qui est statistiquement la plus à même de déclencher la décharge du neurone. En effet, si la décharge est indépendante du stimulus, alors la STA sera nulle parce que les différents segments se neutraliseront dans la moyenne. L'analyse STA constitue, de loin, la forme la plus utilisée de corrélation inverse, et elle a permis de nombreuses avancées en neurosciences sensorielles [24-26].

Cependant l'analyse STA montre ses limites lorsqu'il existe des relations plus complexes entre le stimulus et la réponse, ou lorsque le stimulus pertinent est dans un espace multidimensionnel, comme une scène visuelle ou encore l'ensemble des stimulations tactiles sur une région du corps. Dans ce cas, la STA peut, curieusement, prendre des valeurs proches de zéro sans que ceci signifie nécessairement qu'il n'y a pas de relation entre stimulus et activité neuronale. En effet, l'ensemble des segments de stimulus qui précèdent les PA peut être divisé en sous-ensembles, dont la moyenne de chacun est différente de zéro, alors que la moyenne sur tout l'ensemble pourrait être quasi-nulle. En d'autres termes, il peut exister plusieurs modes symétriques dans le stimulus, et chaque mode peut induire des réponses neuronales. Dans ce cas, il faut utiliser une méthode plus complexe, l'analyse STC (spike-triggered covariance) que nous avons appliquée dans notre étude. L'analyse STC permet de détecter la «dimensionnalité » de la relation stimulus-réponse, c'est-à-dire le nombre minimal de modes ou de dimensions nécessaires pour expliquer la sélectivité du neurone à l'espace sensoriel. Si le nombre de modes est égal à un, alors l'analyse STA suffit en général à caractériser la relation stimulus-réponse. S'il est supérieur à un, alors il faut extraire de l'ensemble des stimulus les différents modes les plus significatifs (ceux dont la relation avec la réponse est la plus forte). C'est ce que réalise l'analyse STC.

Pourquoi la covariance? Intuitivement, lorsque plusieurs modes sont présents, la moyenne peut être nulle, mais la variance sera typiquement altérée, plus grande ou plus petite que la variance qu'on obtiendrait selon les lois des statistiques si le neurone était insensible au stimulus. Si l'on parvient à identifier les différents modes ou dimensions pertinentes, le classement des stimulus en fonction de ces modes permettra de décomposer cette variance, comme étant la somme des variances produites par chaque mode individuellement.

L'application de ces techniques aux stimulus sensoriels (visuels ou tactiles) permet de déterminer quels sont les aspects du stimulus qui sont nécessaires pour expliquer statistiquement la réponse des neurones enregistrés lors de l'expérience ou, en d'autres termes, ce que les neurones « codent » dans le stimulus. Pour l'expliquer quantitativement, on utilise un modèle simplifié du neurone (linear-non linear ou $L N$ ), qui consiste en une combinaison linéaire des différents modes, dont le résultat est comparé à une fonction de décision pour déterminer l'émission d'un PA, ce qui représente une version très simplifiée de la dynamique du potentiel de membrane des neurones. À l'aide de l'analyse STC et des modèles LN, il a été possible de rendre compte des décharges neuronales dans plusieurs systèmes sensoriels, tels que la vision [27-29], le sens tactile [21] ou l'olfaction [30].

des cellules locales s'est avérée très différente. En effet, dans ce cas, ces neurones non seulement répondent à la vibrisse principale, mais aussi aux vibrisses qui l'entourent, à condition que celles-ci aient une direction opposée à la stimulation de la vibrisse principale (Figure 3B). Ce nouveau modèle des cellules locales est capable de rendre compte d'une large part des effets non linéaires que nous avons évoqués précédemment et, par extension, de la réponse à un mouvement global sur toutes les vibrisses.

Le modèle de la réponse fonctionnelle du cortex à tonneaux que nous avons construit durant ces expériences révèle donc l'existence de deux types cellulaires complémentaires, qui mesurent respectivement la corrélation et le contraste spatial présents dans les stimulations multivibrissales appliquées sur les vibrisses du rat. Une propriété remarquable de ces nouveaux types cellulaires est leur aspect dynamique en fonction du stimulus. En particulier, les cellules «locales » effectuent un calcul différent durant les stimulations décorrélées (elles détectent les stimulations sur la vibrisse principale sans se préoccuper des vibrisses du pourtour) et durant les stimulations corrélées (elles détectent alors le contraste entre la vibrisse principale et les vibrisses qui l'entourent).

\section{Conclusion}

Comme nous venons de le voir, un même volume cortical peut contenir de multiples réseaux neuronaux qui adaptent leur stratégie de codage de l'information en fonction des statistiques des entrées sensorielles (voir encadré). Une extrapolation inattendue de ce travail est que, lors de l'interprétation des données issues des techniques d'imagerie fonctionnelle à grande échelle, telles que l'imagerie par résonnance magnétique fonctionnelle (IRMf), on s'appuie souvent sur l'hypothèse qu'il y a une correspondance un-à-un entre l'activation d'une zone cérébrale spécifique et un traitement de l'information bien défini. La description dans notre étude d'un réseau cortical capable de se placer dans des régimes différents de traitement de l'information en fonction du contexte devrait plaider pour une interprétation plus nuancée des résultats issus de l'IRMf [22] grâce à une compréhension plus complète des bases neuronales du signal utilisé. 
En conclusion, le cortex à tonneaux des rongeurs est un modèle de choix pour l'étude des mécanismes neuronaux de la sensation et de la perception tactile. Des stratégies de codage dépendantes des statistiques des entrées sensorielles sont implémentées de manière simultanée dans ce cortex, combinant ainsi dans une seule aire corticale des traitements comparables à ceux effectués par plusieurs aires spécialisées dans le système visuel du chat et des primates. Notre travail de caractérisation fonctionnelle de ces réseaux devrait se poursuivre par la caractérisation cellulaire et anatomique des neurones impliqués à l'aide de méthodes de marquage cellulaire spécifique, par l'imagerie à deux photons et l'optogénétique, ainsi que par la modélisation théorique de ces propriétés de codage de l'information tactile. $\diamond$

\section{SUMMARY}

What the whiskers tell the tactile brain

The rodent whisker system became one of the main system models for the study of the functional properties of sensory neurons. This is due on one hand to the detailed knowledge that we have on the afferent pathways linking the mechanoreceptors in the follicles to the primary somatosensory cortex and on the other hand to the possibility of controlling the sensory input at a micrometer and millisecond scale. The observation of the natural use of the whiskers by rodents indicates that exploration of objects and textures imply multiple contacts with tens of whiskers simultaneously. We have studied the neural code in the barrel cortex, which receives tactile information from the whiskers. By combining multi-electrode recordings and controlled multiwhisker tactile stimulation with theoretical analysis, we have observed a dependence of neural responses on the statistics of the sensory input. Several classes of neuronal responses, similar to those described in a number of cortical visual areas, were observed in the same cortical volume, indicating that various coding schemes are implemented in the same cortical network and can be put into play differentially to cope with the changing statistics of the peripheral stimuli. $\diamond$

\section{LIENS D'INTÉRÊT}

Les auteurs déclarent n'avoir aucun lien d'intérêt concernant les données publiées dans cet article.

\section{REMERCIEMENTS}

Le travail original a été financé par l'Agence nationale de la recherche (NATACS, HR-CORTEX et TRANSTACT) et l'Union européenne (FACETS FP6-015879, BrainScaleS FP7-269921 et Brain-i-Nets FP7-243914). Sami El Boustani a été financé par la Fondation pour la recherche médicale. Dan Shulz a occupé une chaire de Professeur invité (Weston visiting professorship) à l'Institut Weizmann (Israël) pendant la préparation de ce manuscrit.

\section{RÉFÉRENCES}

1. Geldard FA, Sherrick CE. The cutaneous rabbit: a perceptual illusion. Science $1972 ; 178$ : 178-9.

2. Blankenburg F, Ruff CC, Deichmann R, et al. The cutaneous rabbit illusion affects human primary sensory cortex somatotopically. PLoS Biol $2006 ; 4$ : e69.

3. Miyazaki M, Hirashima M, Nozaki D. The cutaneous rabbit hopping out of the body. J Neurosci $2010 ; 30: 1856-60$.

4. Gao P, Bermejo R, Zeigler PH. Whisker deafferentation and rodent whisking patterns: behavioral evidence for a central pattern generator. J Neurosci 2001 ; 21 : 5374-80.
5. Woolsey TA, van der Loos H. The structural organization of layer IV in the somatosensory region (SI) of mouse cerebral cortex. The description of a cortical field composed of discrete cytoarchitectonic units. Brain Res 1970 ; $17: 205-42$.

6. Killackey HP, Belford GR. The formation of afferent patterns in the somatosensory cortex of the neonatal rat. J Comp Neurol 1979 ; 183 : 285 303.

7. Simons DJ. Temporal and spatial integration in the rat SI vibrissa cortex. J Neurophysiol $1985 ; 54: 615-35$.

8. Carvell GE, Simons DJ. Biometric analyses of vibrissal tactile discrimination in the rat. J Neurosci $1990 ; 10: 2638-48$.

9. Ritt JT, Andermann ML, Moore Cl. Embodied information processing: vibrissa mechanics and texture features shape micromotions in actively sensing Rats. Neuron 2008 ; 57 : 599-613.

10. Moore $\mathrm{Cl}$, Nelson SB. Spatio-temporal subthreshold receptive fields in the vibrissa representation of rat primary somatosensory cortex. J Neurophysiol $1998 ; 80: 2882-92$.

11. Zhu JJ, Connors BW. Intrinsic firing patterns and whisker evoked synaptic responses of neurons in the rat barrel cortex. J Neurophysiol 1999; 81 : 1171-83.

12. Erchova I, Jacob V, Ego-Stengel V, et al. Multiwhisker suppressive interactions in the rat barrel cortex described by a MAX operator. Soc Neurosci Abstract 2006 ; 144 : 17.

13. Petersen RS, Diamond ME. Spatial-temporal distribution of whisker-evoked activity in rat somatosensory cortex and the coding of stimulus location.J Neurosci $2000 ; 20: 6135-43$.

14. Mirabella G, Battiston S, Diamond ME. Integration of multiple-whisker inputs in rat somatosensory cortex. Cereb Cortex $2001 ; 11: 164-70$.

15. Bringuier V, Chavane F, Glaeser L, et al. Horizontal propagation of visual activity in the synaptic integration field of area 17 neurons. Science 1999 ; $283: 695-9$.

16. Shimegi S, Ichikawa T, Akasaki T, et al. Temporal characteristics of response integration evoked by multiple whisker stimulations in the barrel cortex of rats. J Neurosci $1999 ; 19: 10164-75$.

17. Ego-Stengel V, Souza TM, Jacob V, et al. Spatiotemporal characteristics of neuronal sensory integration in the barrel cortex of the rat. J Neurophysiol $2005 ; 93: 1450-67$.

18. Jacob V, Le Cam J, Ego-Stengel V, et al. Emergent properties of tactile scenes selectively activate barrel cortex neurons. Neuron $2008 ; 60: 1112-25$.

19. Jacob V, Estebanez L, Le Cam J, et al. The matrix: a new tool for probing the whisker-to-barrel system with natural stimuli. J Neurosci Methods 2010 ; $189: 65-74$.

20. Ego-Stengel V, Le Cam J, Shulz DE. Coding of apparent motion in the thalamic nucleus of the rat vibrissal somatosensory system. J Neurosci $2012 ; 32: 3339-51$.

21. Estebanez L, El Boustani S, Destexhe A, et al. Correlated input reveals coexisting coding schemes in a sensory cortex. Nat Neurosci $2012 ; 12$ : 1691-99.

22. Op de Beeck HP, Haushofer J, Kanwisher NG. Interpreting fMRI data: maps, modules and dimensions. Nat Rev Neurosci 2008 ; 9 : 123-35.

23. Geldard, FA. Saltation in somesthesis. Psychol Bull $1982 ; 92: 136-75$.

24. Jones JP, Palmer LA. The two-dimensional spatial structure of simple receptive fields in cat striate cortex. J Neurophysiol 1987 ; 58 : 1187-211.

25. DeAngelis GC, Ohzawa I, Freeman RD. Receptive-field dynamics in the central visual pathways. Trends Neurosci $1995 ; 18: 451-8$.

26. Jenison RL, Schnupp JW, Reale RA, et al. Auditory space-time receptive field dynamics revealed by spherical white-noise analysis. J Neurosci $2001 ; 21$ : 4408-15.

27. Ringach DL. Mapping receptive fields in primary visual cortex. J Physiol $2004 ; 558: 717-28$.

28. Rust NC, Schwartz 0 , Movshon JA, et al. Spatiotemporal elements of macaque vl receptive fields. Neuron $2005 ; 46: 945-56$.

29. Schwartz 0, Pillow JW, Rust NC, et al. Spike-triggered neural characterization. J Vision $2006 ; 6$ : 484-507.

30. Geffen MN, Broome BM, Laurent $G$, et al. Neural encoding of rapidly fluctuating odors. Neuron $2009 ; 61: 570-86$.
TIRÉS À PART

D.E. Shulz 